\title{
Erratum: Axion stars in the infrared limit
}

\section{Joshua Eby, Peter Suranyi, Cenalo Vaz and L.C.R. Wijewardhana}

Dept. of Physics, University of Cincinnati, Cincinnati, $\mathrm{OH}$ 45221, U.S.A.

E-mail: joshaeby@gmail.com, peter.suranyi@uc.edu, cenalo.vaz@uc.edu, rohana.wijewardhana@gmail.com

ERRATUM TO: JHEP03(2015)080

ARXIV EPRINT: 1412.3430

In eq. (2.5), the first expectation value should read $2 N R(r)^{2}$ rather than $2 N^{2} R(r)^{2}$. The correct expression was used in the calculations.

In eq. (2.7), there is an errant minus sign on the right hand side of the second equation; it should read

$$
\frac{B^{\prime}}{A B r}-\frac{A-1}{A r^{2}}=\frac{f_{a}^{2}}{M_{P}^{2}}\left[\frac{E^{2} N R^{2}}{B f_{a}^{2}}+\frac{N R^{\prime 2}}{A f_{a}^{2}}-m^{2}\left[1-J_{0}(X)\right]\right] .
$$

The correct expression was used in the calculations.

In eq. (3.1), the first equality has an errant factor of 3 in the denominator, and should read

$$
M=-4 \pi \int_{0}^{\infty} T_{00} r^{2} d r
$$

As a result, eq. (3.2) contains the wrong prefactor as well; it should read

$$
V(y)=\pi \int_{0}^{y} Y(x)^{2} x^{2} d x .
$$

This error was carried into the calculations of the total mass, and causes us to overestimate the masses in table 1 by a consistent factor of $3 / 2$. A corrected version of table 1 and figure 2 are below: 


\begin{tabular}{|l|l|l|l|l|}
\hline$\lambda$ & $M(\mathrm{~kg})$ & $R_{99}(\mathrm{~km})$ & $\mathrm{d}\left(\mathrm{kg} / \mathrm{m}^{3}\right)$ & $\delta M(\mathrm{~kg})$ \\
\hline 0.1 & $1.00 \times 10^{18}$ & 115 & 155 & 125000. \\
0.3 & $3.45 \times 10^{18}$ & 386 & 14.3 & 46300. \\
0.4 & $5.08 \times 10^{18}$ & 593 & 5.81 & 33500. \\
0.5 & $6.33 \times 10^{18}$ & 854 & 2.43 & 16300. \\
0.54 & $6.56 \times 10^{18}$ & 972 & 1.70 & 8350. \\
0.58 & $6.63 \times 10^{18}$ & 1076 & 1.27 & 1170. \\
0.62 & $6.61 \times 10^{18}$ & 1183 & .951 & -5370. \\
0.8 & $5.98 \times 10^{18}$ & 1652 & 0.317 & -23200. \\
1 & $5.14 \times 10^{18}$ & 2145 & 0.124 & -33100. \\
2 & $2.78 \times 10^{18}$ & 4499 & 0.00729 & -53400. \\
4 & $1.42 \times 10^{18}$ & 9062 & 0.000457 & -88700. \\
10 & $5.74 \times 10^{17}$ & 22849 & 0.0000115 & -267000. \\
\hline
\end{tabular}

Table 1. Mass, radius, average density, and $\delta M$, as defined in (3.13), as a function of $\lambda=$ $f_{a} /\left(M_{P} \Delta\right)$.

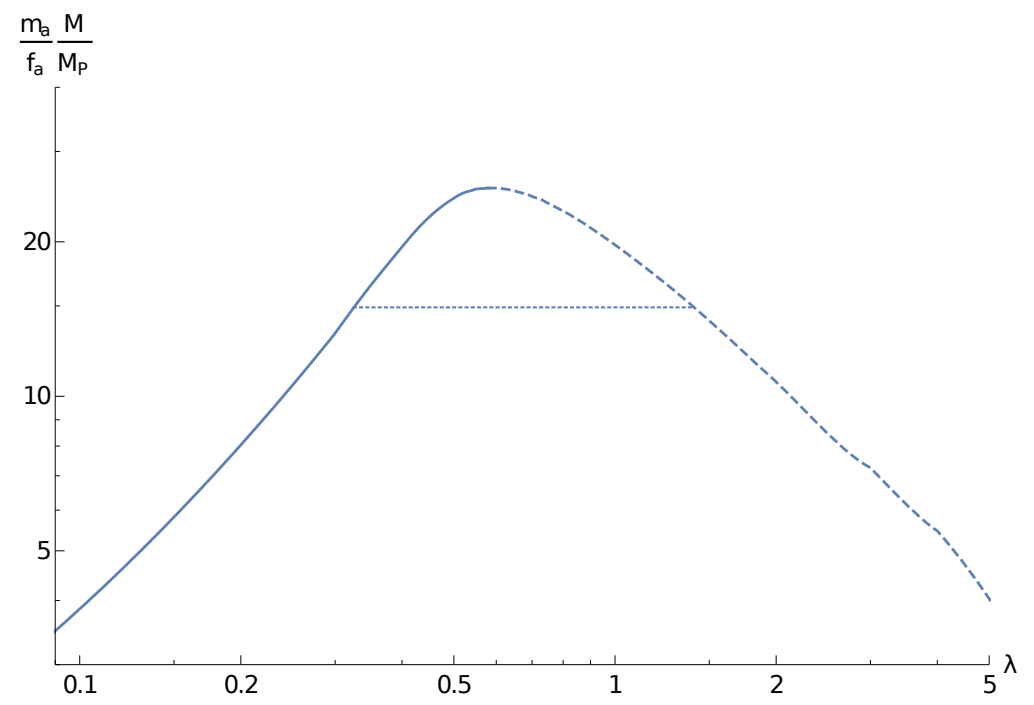

Figure 2. The mass $M$, scaled by the factor $m_{a} /\left(f_{a} M_{P}\right)$, as a function of $\lambda$. Stable (unstable) stars are represented by the solid (dashed) line. The dotted line connects sample states with identical numbers of axions. The mass difference of these stars is a small fraction of the mass.

Open Access. This article is distributed under the terms of the Creative Commons Attribution License (CC-BY 4.0), which permits any use, distribution and reproduction in any medium, provided the original author(s) and source are credited. 